\title{
Dynamic Proxy Tree-Based Data Dissemination Schemes for Wireless Sensor Networks
}

\author{
Wensheng Zhang, Guohong Cao, and Tom La Porta \\ Department of Computer Science and Engineering \\ The Pennsylvania State University \\ University Park, PA 16802 \\ E-mail: \{wezhang, gcao, tlp $\} @$ cse.psu.edu
}

\begin{abstract}
In wireless sensor networks, efficiently disseminating data from a dynamic source to multiple mobile sinks is important for applications such as mobile target detection and tracking. The tree-based multicasting scheme can be used. However, due to the short communication range of each sensor node and the frequent movement of sources and sinks, a sink may fail to receive data due to broken paths, and the tree should be frequently reconfigured to reconnect sources and sinks. To address the problem, we propose a dynamic proxy tree-based framework in this paper. A big challenge in implementing the framework is how to efficiently reconfigure the proxy tree as sources and sinks change. We model the problem as on-line constructing a minimum Steiner tree in an Euclidean plane, and propose centralized schemes to solve it. Considering the strict energy constraints in wireless sensor networks, we further propose two distributed on-line schemes, a shortest path-based (SP) scheme and $a$ spanning range-based (SR) scheme. Extensive simulations are conducted to evaluate the schemes. The results show that the distributed schemes have similar performance as the centralized ones, and among the distributed schemes, SR outperforms SP.
\end{abstract}

\section{Introduction}

A wireless sensor network [2] consists of many tiny and inexpensive sensor nodes that are distributed over a vast field to obtain sensing data. These nodes are capable of not only measuring real world phenomena, but also storing, processing and transferring these measurements. Due to these attractive characteristics, sensor networks are adopted in many military and civil applications such as battlefield surveillance, environmental control, and security management. In these applications, sensing data usually need to be disseminated from a source to many sinks, where the source and the sinks may frequently move. For example, a sensor network may be deployed in a battlefield to detect and monitor the enemy tanks and soldiers. When such a target is detected, the sensing data about the target (e.g., its location, velocity and the geographic characteristics of its surrounding area) should be sent to commanders and soldiers, who may also move in the battlefield.

In recent years, many data dissemination schemes [11, $13,21,19,8,9,23]$ have been proposed for sensor networks, but most of them can not efficiently support multicasting from a dynamic source to multiple mobile sinks. For example, the external storage-based scheme [11], the datacentric storage-based (DCS) scheme [19] and the indexbased scheme [23] only consider the point-to-point communication between a pair of source and sink. The directed diffusion scheme [13] and the two-tier data dissemination (TTDD) scheme [21] naturally support data multicasting, but they are not efficient when the source and the sinks are mobile. In the directed diffusion scheme, a source needs to flood availability information over the whole network. Even though the flooding rates at different areas can be adaptively changed according to the locations and the query rates of the sinks, there still exist lots of redundancy. The TTDD scheme proactively maintains a grid-based propagation structure over the whole network in spite of the actual locations of the sinks, and the structure should be updated whenever the source location changes, which may cause high maintenance overhead.

To avoid unnecessarily flooding information [13] or expanding propagation structure over the whole network [21], the tree-based multicasting scheme can be used. In this scheme, the source and the sinks form a tree rooted at the source, and the source pushes data to the sinks along the tree branches. However, due to the short communication range of each sensor node and the frequent movement of sources and sinks, a sink may frequently fail to receive data due to broken paths, and the tree should be frequently re- 
configured to reconnect sources and sinks. To address the problems, we propose a dynamic proxy tree-based framework. In this framework, each source (sink) is associated with a stationary sensor node called source (sink) proxy. The proxies related to the same source form a proxy tree. Facilitated by the tree, a source can push data to its proxy, which further pushes the data to multiple sink proxies, and a sink can query its proxy to get the data.

As a source changes or a sink moves, the associated proxy should be changed to reduce the cost of pushing (querying) data to (from) the proxy. Accordingly, the tree should also be reconfigured to reduce the cost of multicasting data from the source proxy to the sink proxies. Due to the strict energy constraints in sensor networks, tree reconfiguration should be conducted in an energy efficient way. Many multicasting tree reconfiguration schemes have been proposed for the existing wired and wireless networks such as the Internet, the cellular network and the wireless ad hoc network. However, these schemes can not be directly applied to the wireless sensor network due to their large overhead. For example, in the rearrangeable inexpensive edgebased on-line steiner (ARIES) algorithm [3], a new node joins an existing tree via the shortest path to the tree, and the subtrees including newly added or deleted nodes are reconfigured every certain time. This algorithm requires each multicasting member to know its distance to other members. It is suitable for the Internet and the cellular network, in which each router (base station) can naturally obtain the information through the underlying topology advertisement protocol (e.g., OSPF). However, it is not suitable for sensor networks, where running the topology advertisement protocol may cause large overhead. On the other hand, the multicasting protocols $[16,17,10]$ for mobile ad hoc networks emphasize more on route robustness and pay less attention to energy efficiency, since mobile ad hoc networks have frequent path breaks due to high node mobility.

In this paper, we first formalize the tree reconfiguration problem as an on-line Euclidean steiner problem [18], and propose several Voronoi diagram-based centralized schemes to solve the problem. Considering the strict energy constraints and the locality requirements in wireless sensor networks, we propose two distributed heuristicbased schemes, the shortest path-based (SP) scheme and the spanning range-based (SR) scheme. These schemes are motivated by the following observations: First, the new proxy of a source (sink) can utilize the information provided by the previous proxy to efficiently join the tree. Second, localized adjustments can be conducted at individual nodes to gradually optimize the tree structure. With the SP scheme, when a sink (source) changes its proxy, the new proxy uses flooding to join the tree. The proxy changes also cause the tree nodes to gradually adjust their locations in a localized way. Since SP still has large overhead due to flooding, es- pecially when the tree nodes are far away from each other, the SR scheme is proposed. In this scheme, each subtree is associated with a certain spanning range, which is dynamically assigned and adjusted. With a few messages, a new proxy can find the root of the smallest subtree whose spanning range covers itself, and joins the subtree.

We use extensive simulations to compare the proposed schemes in terms of data dissemination cost and tree reconfiguration overhead. The results show that the centralized schemes slightly outperform the distributed schemes, and the SR scheme outperforms the SP scheme.

The rest of the paper is organized as follows: Section II describes the system model and the dynamic tree-based framework. Section III proposes centralized schemes for tree reconfiguration. The distributed schemes are presented in Section IV. Section V reports the performance evaluation results, and Section VI finally concludes the paper.

\section{Preliminaries}

\subsection{System Model}

We consider a wireless sensor network that consists of many stationary sensor nodes. These nodes are densely deployed over a vast field to detect and continuously monitor some mobile targets. The network is connected, and the field can be completely sensed. Each sensor node knows its own location through GPS [1] or other inexpensive techniques such as triangulation [5]. Based on the location information, some location-based routing protocols [4, 15] can be used for multi-hop communication between sensor nodes.

Some mobile hosts (e.g., PDAs) are moving within the sensing field. They can query and receive sensing data from sensor nodes. A mobile host can directly communicate with a sensor node if it is within the transmission range of the node. For simplicity, we do not consider the communication between the mobile hosts.

When a mobile target of interest appears in the sensing field, the sensor nodes surrounding it can detect it and the leader (source) may exclusively detect the target [6] or aggregate the related detections [22], periodically generates sensing data about the target, and disseminates the data to some mobile hosts (sinks) that have subscribed for the sensing data. As the target moves away from its current source, the source is changed to be another node closer to the target.

To facilitate a sink finding a source of interest, the indexbased scheme proposed in [23] is adopted. In this scheme, some index nodes maintain the locations of sources, and a sink can query the appropriate index nodes to get the location of a source. When a source is changed, its location is updated at the related index nodes, such that the sinks can still find it. 


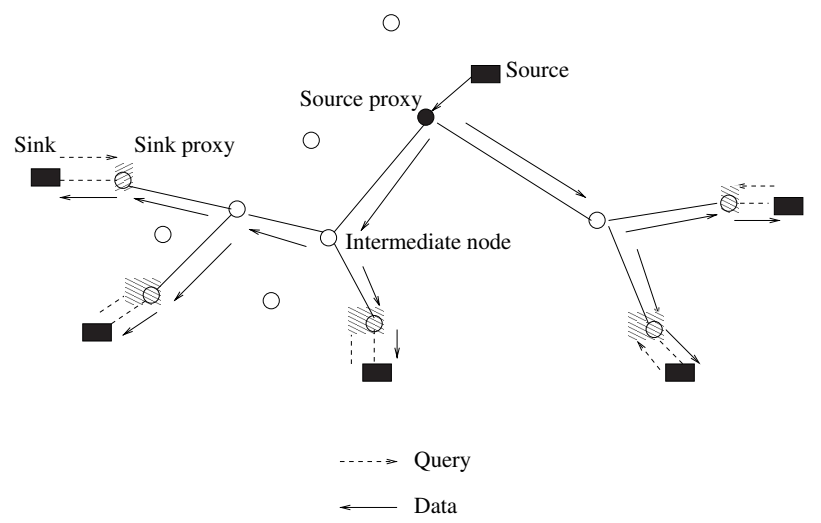

Figure 1. Using proxy tree to support dynamic multicasting

\subsection{Dynamic Proxy Tree-Based Framework}

Due to the dynamic characteristics of sources and sinks, it is difficult to maintain a tree that directly connects a source and multiple sinks that are interested in the source, or disseminate data directly from the source to the sinks. To deal with the problem, we propose a proxy tree-based framework. In the framework, as shown in Figure 1, a source (sink) is associated with a stationary sensor node called source (sink) proxy. As the location of the source (sink) changes, its proxy does not change until its distance to the source (sink) exceeds a certain threshold. A source proxy and the proxies of the sinks that need to frequently query the source form a proxy tree. Facilitated by the tree, sensing data is periodically pushed from the source to its proxy, and then is multicast to sink proxies in the tree. Each sink can query data from its proxy. The change of a source (sink) proxy may cause the proxy tree to be reconfigured to reduce the cost for pushing data from the source proxy to the sink proxies and from sink proxies to sinks. In the remaining of the paper, we focus on efficiently reconfiguring the proxy tree to minimize the data dissemination cost and the tree reconfiguration overhead.

\section{Centralized Tree Reconfiguration Schemes}

The problem of forming a minimum-cost proxy tree can be formalized as constructing a minimum Steiner tree [14] that connects a given set of terminals in a graph. Due to the dense deployment of sensor nodes, we can further formalize the problem as constructing a minimum Steiner tree in an Euclidean plane. In this section, we describe a centralized off-line scheme and several centralized on-line schemes to address the problem.

\subsection{An Off-Line Scheme}

Constructing a minimum Steiner tree is known as a NPhard problem [12], and the exact solution of the problem has very high computational complexity. Heuristic-based solutions have been proposed to solve the problem, and many of them are based on the idea of optimizing the minimum spanning tree to approach a minimum steiner tree. Next, we present an off-line scheme for constructing an approximated minimum-cost proxy tree, which is similar to the algorithm proposed by Smith et al. [20].

The scheme makes use of the observation that a minimum steiner tree (minimum-cost proxy tree) is a union of full Steiner trees (FSTS), and each FST is a tree with the following properties:

- It spans $k(k>1)$ terminals (proxies) and has $k-2$ steiner points.

- Each Steiner points has three edges making $120^{\circ}$ with each other, and every proxy in the FST has degree one.

Based on the above observation, a minimum-cost proxy tree is constructed in two steps: first, a minimum spanning tree (denoted as $T$ ) including all the proxies is constructed; second, $T$ is reconfigured to be a set of FSTs. The Kruskal's algorithm [7] can be used to construct $T$, and the procedure for reconfiguring $T$ is described as follows:

1. $T$ is decomposed into multiple components, each with $i(i=2,3, \cdots, m)$ proxies, where a 2-proxy component is an edge of $T$, and an $i$-proxy $(i>2)$ component is a corner that has $(i-1)$ edges.

2. For each $i$-proxy component $T_{i}$, a FST (denoted as $F S T\left(T_{i}\right)$ ) that consists of all the $i$ proxies is constructed. All the generated FSTs are placed on a priority queue $Q$ based on the value of

$$
\left|F S T\left(T_{i}\right)\right| /\left|T_{i}\right| .
$$

3. An approximated minimum-cost proxy tree is constructed by picking FSTs from $Q$ in the same way as the Kruskal's algorithm.

\subsection{On-Line Schemes}

When a sink joins (leaves) a multicasting group, or moves far away from its current proxy, the proxy set has to be changed by adding (removing) a proxy, and the tree should also be reconfigured to reduce the data dissemination cost. Since it is overly expensive to totally reconstruct the tree after each membership change, we borrow the idea of ARIES [3] and propose an approximated on-line minimum steiner tree (ONMST) scheme. 
In ONMST, a new proxy (denoted as $P_{n}$ ) is added to the current proxy tree in two steps: first, $P_{n}$ is added to the current tree via the shortest path that connects the tree and $P_{n}$; second, a small subtree that contains $P_{n}$ is optimized based on the locality property of the Voronoi diagram [14]. Specifically, the procedure is described as follows:

1. The current proxy tree (denoted as $T_{c}$ ) is divided into multiple Voronoi cells.

2. Suppose $P_{n}$ is covered by the Voronoi cell of node $P_{i}$. We construct a node set $\mathcal{Y}$ which includes $P_{n}$ and each node that is either a vertex of the Voronoi cell or a neighbor of a vertex of the cell.

3. In the subgraph (denoted as $G_{s}$ ) of $T_{c}$ which contains nodes in $\mathcal{Y}$, the off-line scheme presented in Section 3.1 is used to construct one or more approximated Steiner trees. Note that, in the tree(s), a pair of nodes is connected if and only if they are connected in $T_{c}$ (except that $P_{n}$ is connected with $P_{i}$ ). The reconfigured subgraph is denoted as $G_{s}^{\prime}$.

4. $T_{c}$ is replaced by a new tree $T_{c}^{\prime}$, which is obtained by replacing $G_{s}$ with $G_{s}^{\prime}$.

When a proxy should leave the current tree, it is removed only if it is a leaf.

The ONMST scheme can be further optimized by letting each Steiner node (denoted as $P_{i}$ ) on the tree to adjust its location every certain time interval. Let $\mathcal{Y}$ denote a node set containing the neighbors of $P_{i}$. A FST that consists of the nodes in $\mathcal{Y}$ is computed, and $P_{i}$ is replaced by the newly introduced Steiner nodes in the FST. In the following, we call the enhanced ONMST scheme E-ONMST.

\section{Distributed Tree Reconfiguration Schemes}

Even though the ONMST scheme and the E-ONMST scheme have lower complexity than the off-line scheme, they may not be suitable for sensor networks due to the following reasons: Each sensor node has only partial knowledge of the multicasting group; i.e., it only knows its neighbors in the tree. When a proxy changes from one node to another, requiring the new proxy or its neighbor to collect necessary information to construct Voronoi diagram and reconfigure the subgraph surrounding itself may cause large overhead. To address the problem, we propose two distributed heuristic-based schemes in this section.

\subsection{Shortest Path-Based (SP) Scheme}

The SP scheme is based on the heuristic that a new proxy $\left(P_{n}\right)$ should join the current proxy tree by attaching to the tree node $\left(P_{i}\right)$ that has the shortest distance to it. $P_{i}$ then

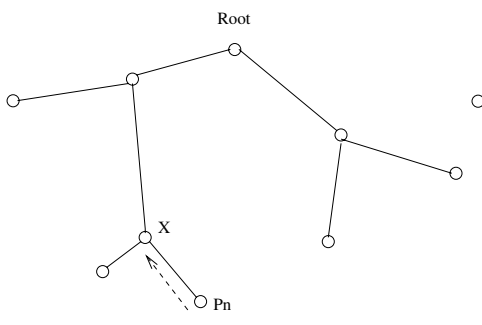

(a)

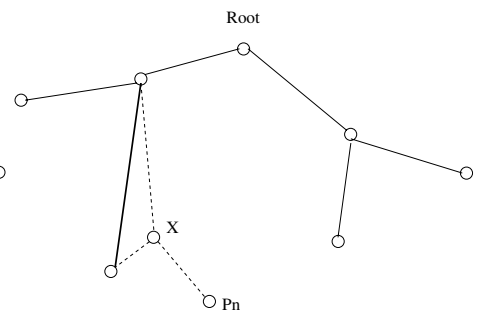

(b)
Figure 3. A node leaves the tree.

conducts localized reconfigurations within the subtree containing itself and its neighbors. Also, each node periodically conduct localized reconfiguration to gradually optimize the tree.

\subsubsection{Proxy Join and Leave}

When a sink wants to join the proxy tree, it selects a nearby sensor node $\left(P_{n}\right)$ as its proxy. $P_{n}$ joins the tree by going through the following three steps.

Step 1: Pre-searching. $P_{n}$ obtains the location of the current source proxy (root) from the appropriate index nodes (refer to Section 2), and then sends a join_req to the root. On receiving the request, as shown in Figure 2 (a), the root forwards the request to its neighbor that is closest to $P_{n}$, and the neighbor further forwards the request to its own neighbor that is closest to $P_{n}$. The forwarding procedure continues, until it reaches a node $\left(P_{j}\right)$ which is closer to $P_{n}$ than any of its neighbors, and a message join_rep is sent from $P_{j}$ to $P_{n}$.

Step 2: Finding the closest node. On receiving the reply, as shown in Figure 2 (b), $P_{n}$ floods a message discover within a circle that is centered at itself and has a radius of $d_{P_{n}, P_{j}}$ (i.e., the distance between $P_{i}$ and $P_{j}$ ). Every node receiving the discover replies its location to $P_{n}$. Based on the replies, $P_{n}$ finds the node $\left(P_{i}\right)$ which is closest to itself, and sends a confirm message to $P_{i}$.

Step 3: Node join. On receiving the confirm message, as shown in Figure 2 (c), $P_{i}$ adds in $P_{n}$, and reconfigures the subtree containing itself and its neighbors into a FST.

When $P_{n}$ wants to leave, and it is a leaf in the tree, as shown in Figure 3 (a), it leaves the tree and sends a leave_req to its parent. Otherwise, $P_{n}$ has to stay in the tree and mark itself as a Steiner node. On receiving leave_req, the parent node removes $P_{n}$. If the parent is a Steiner point and has only two neighbors in the tree, as shown in Figure 3 (b), it removes itself and lets its neighbors directly connect with each other. 


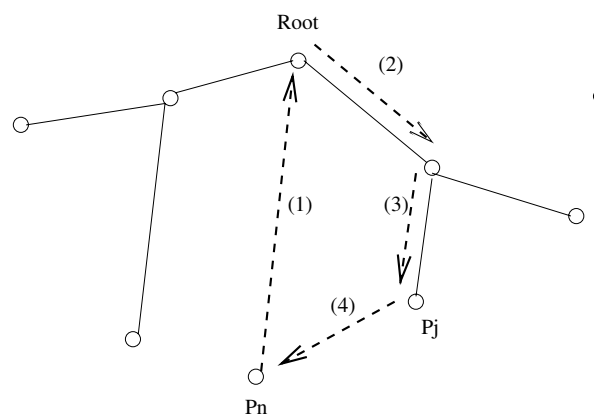

(a)

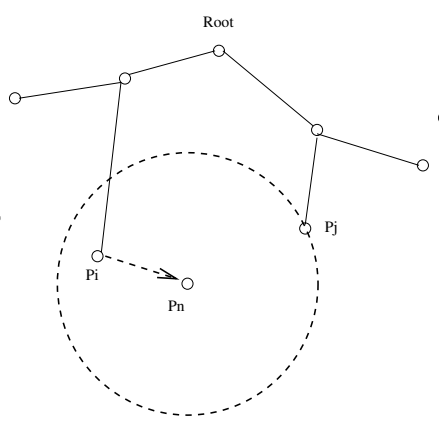

(b)

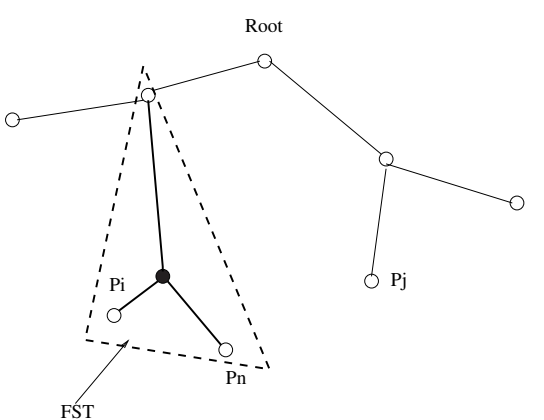

(c)

Figure 2. A new node joins the tree: (a) Pre-searching; (b) Finding the closest node; (c) Joining the node.

\subsubsection{Sink (Source) Movement Initiated Tree Reconfig- uration}

As a sink (source) moves and becomes far away from its proxy, the current proxy $\left(P_{n}\right)$ should be changed to another node $\left(P_{n}^{\prime}\right)$ which is closer to the sink (source). The tree reconfiguration initiated by a proxy change goes through the following three steps.

Step 1: Establishing a temporary edge. As shown in Figure 4 (a), $P_{n}^{\prime}$ sends a migrate_req to $P_{n}$. On receiving the message, $P_{n}$ establishes a temporary edge between $P_{n}^{\prime}$ and its parent (denoted as $X$ ), and leaves the tree.

Step 2: Finding the closest node. As shown in Figure 4 (b), this step is similar to the Step 2 of the new proxy joining procedure. If the found closest node $\left(P_{i}\right)$ is not $X, P_{n}^{\prime}$ tears down the temporary connection with $X$, and attaches to $P_{i}$.

Step 3: Joining the tree. As shown in Figure 4 (c), this step is the same as the Step 3 of the new proxy joining process.

\subsubsection{Periodic Localized Tree Reconfiguration}

When a proxy moves, as shown in Figure 4, the subtrees that it leaves or joins are reconfigured, but the remaining part of the tree is untouched even after it has been affected by the reconfigurations. To address the problem, we propose a periodic localized tree reconfiguration mechanism. With this mechanism, each Steiner point node monitors the changes of its neighbors. Every certain time, it computes the FST of the subgraph including its neighbors and finds the optimal location for itself. If the cost difference between transmitting data via the new FST and via the current subtree exceeds a certain percentage $(\alpha)$, the node replaces itself with the node closest to the calculated optimal Steiner point. With the periodic localized reconfiguration scheme, the tree can be gradually reconfigured with low cost.

\subsection{Spanning Range-Based (SR) Scheme}

In the SP scheme, a new proxy needs to flood discover messages to find its position in the proxy tree. The flooding overhead can be large, especially when the multicasting members are far away from each other. To deal with the drawback, we propose a spanning range-based (SR) scheme. The basic idea of SR is illustrated in Figure 5. As shown in Figure 5 (a), each subtree is assigned a certain spanning range, and the nodes in the subtree tree should be within the range. If a proxy $\left(P_{n}\right)$ in a subtree $\left(P_{i}\right)$ is changed to another one $\left(P_{n}^{\prime}\right)$, as shown in Figure $5(\mathrm{~b}), P_{n}^{\prime}$ should leave subtree $P_{i}$ and join subtree $P_{j}$. During this process, both subtrees should be reconfigured. In the following, we first present the strategy to assign spanning ranges, and then present the algorithms for adding (removing) a proxy and dealing with source (sink) changes.

\subsubsection{Spanning Range Assignment}

Let $P$ be the root of the tree, and $P_{i}(i=0, \cdots, m-1)$ be the children of $P$. As shown in Figure 6 (a), the spanning range for each $P_{i}$ is the halfplane that does not cover $P$ and is confined by the following three lines:

- $l_{i}^{0}$, which passes $P_{i}$ and is perpendicular with line $P P_{i}$;

- $l_{i}^{1}$, which equally divides $\angle P_{i-1} P P_{i}$;

- $l_{i}^{2}$, which equally divides $\angle P_{i} P P_{i+1}$.

Here, $P_{i-1}\left(P_{i+1}\right)$ is the anti-clockwise (clockwise) neighboring sibling of $P_{i}$.

For a node $P_{i, j}$ whose parent nodes is $P_{i}$, as shown in Figure 6 (b), its spanning range is decided as follows:

case 1: $P_{i, j}$ is the most anti-clockwise child of $P_{i}$ (e.g., $P_{1,0}$ in Figure 6(b)). It is confined by

- $l_{i, j}^{0}$, which passes $P_{i, j}$ and is perpendicular with line $P_{i} P_{i, j}$; 


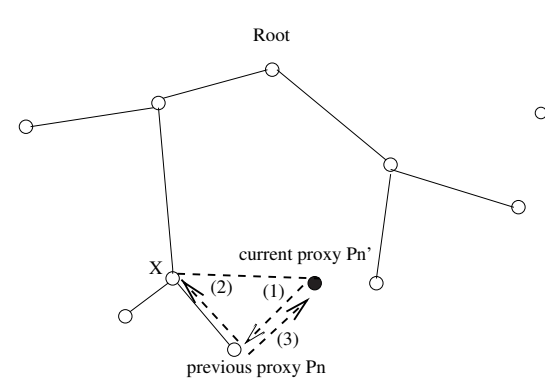

(a)

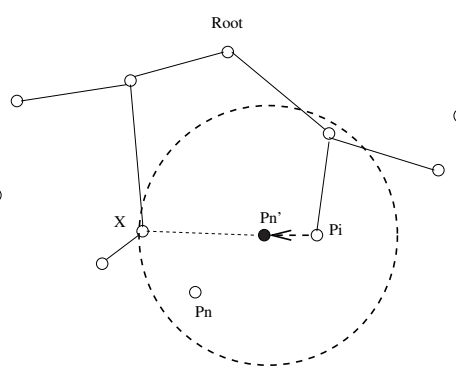

(b)

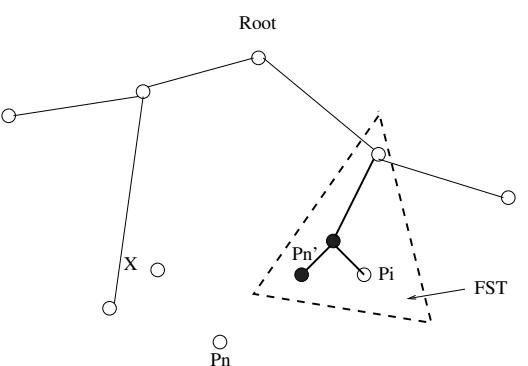

(c)

Figure 4. Sink (source) movement initiated tree reconfiguration

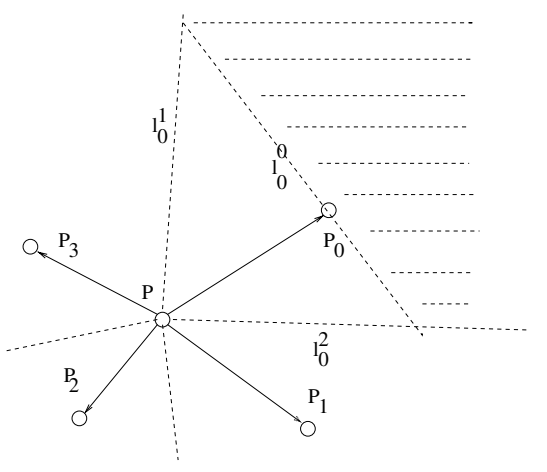

(a)

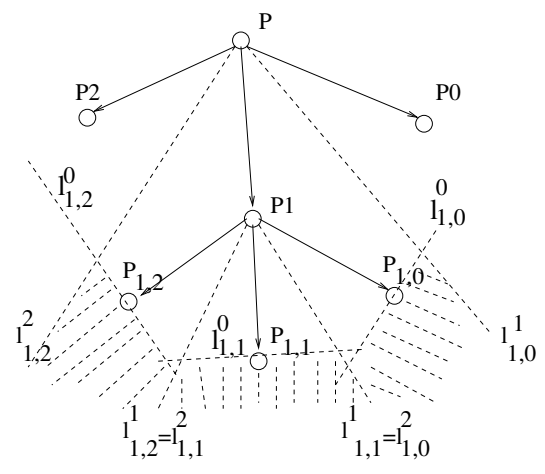

(b)

Figure 6. Spanning ranges of nodes

- $l_{i, j}^{1}$, which equally divides $\angle P_{i-1} P P_{i}$;

- $l_{i, j}^{2}$, which equally divides $\angle P_{i, j} P_{i} P_{i, j+1}$.

case 2: $P_{i, j}$ is the most clockwise child of $P_{i}$ (e.g., $P_{1,2}$ in Figure 6(b)). It is confined by

- $l_{i, j}^{0}$, which is defined before;

- $l_{i, j}^{1}$, which equally divides $\angle P_{i, j-1} P_{i} P_{i, j}$;

- $l_{i, j}^{2}$, which equally divides $\angle P_{i} P P_{i+1}$.

case 3: otherwise (e.g., $P_{1,1}$ in Figure 6(b)). It is confined by

- $l_{i, j}^{0}$, which is defined before;

- $l_{i, j}^{1}$, which equally divides $\angle P_{i, j-1} P_{i} P_{i, j}$;

- $l_{i, j}^{2}$, which equally divides $\angle P_{i, j} P_{i} P_{i, j+1}$.

According to the spanning range assignment rule, each node on the tree can decide the spanning range of its children, and send the range to them. To reduce the overhead, the range information can be piggybacked in data packets sent from the node to its children.

\subsubsection{Node Join}

When a mobile sink wants to join the multicasting tree, similar to the SP scheme, it selects a nearby sensor node $P_{n}$ as its proxy and asks $P_{n}$ to join the tree. $P_{n}$ obtains the current location of the source proxy from some appropriate index nodes, and then sends a join_req to the source proxy $(P)$. On receiving the request, $P$ decides the location of $P_{n}$ as follows:

(1) $P$ calculates the spanning ranges of its children. If $P_{n}$ is covered by the spanning range of a child $P_{i}, P$ forwards join_req $\left(P_{n}\right)$ to $P_{i}$.

(2) Otherwise, $P$ adds $P_{n}$ as its child. In order to add $P_{n}$ at an appropriate position, $P$ first finds a child $P_{j}$, such that $\angle P_{n} P P_{j}$ is no larger than $\angle P_{n} P P_{i}(i=$ $0, \cdots, m-1)$.

(2.1) If $\angle P_{n} P P_{j}<120^{\circ}$, then a FST for triangle $P-P_{n}-P_{j}$ is calculated and replaces the subgraph containing $P, P_{n}$ and $P_{j}$.

(2.2) Otherwise, $P_{n}$ is directly added as a child of $P$.

On receiving a join_req $\left(P_{n}\right)$ forwarded by its parent, $P_{i}$ follows the same procedure as its parent to decide whether to add $P_{n}$ as its child or to forward the join_req to one of 


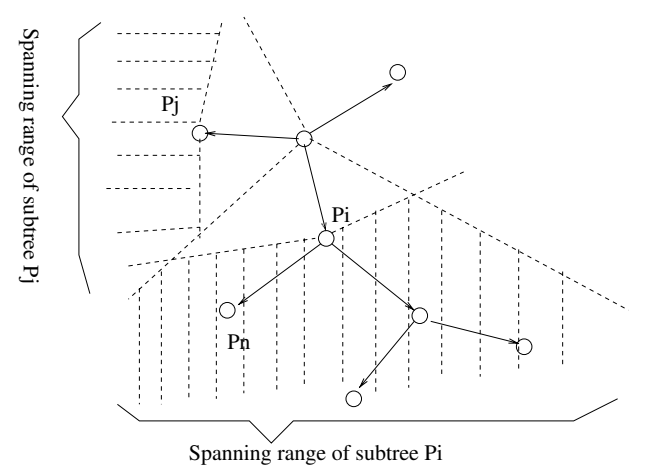

(a)

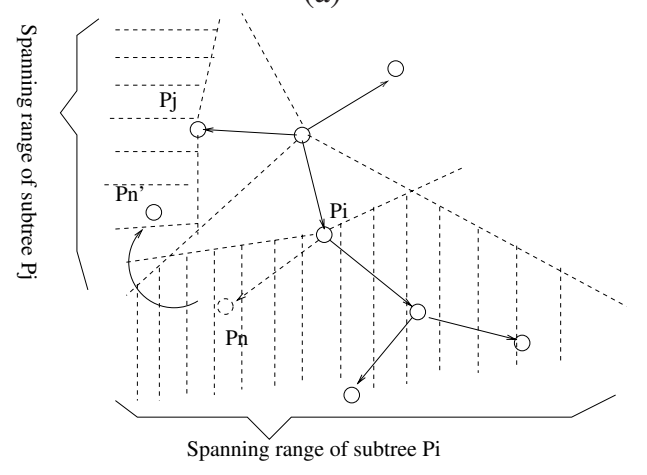

(b)

Figure 5. The basic idea of the SR scheme

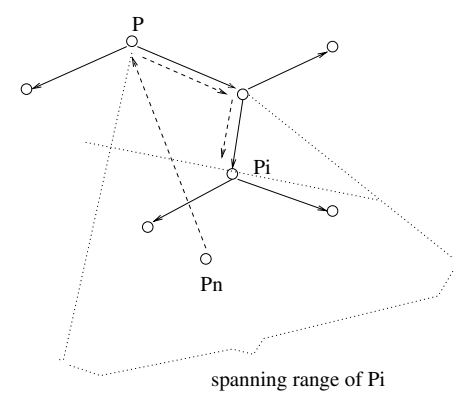

(a)

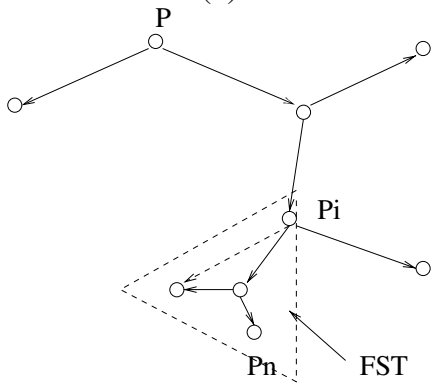

(b)

Figure 7. Adding a new proxy $\left(P_{n}\right)$ its children. The process continues until $P_{n}$ joins the tree. Figure 7 shows an example of adding a new proxy.

\subsubsection{Sink Movement-Triggered Tree Reconfiguration}

As a sink moves and becomes far away from its current proxy $\left(P_{n}\right), P_{n}$ should be changed to another node $\left(P_{n}^{\prime}\right)$ which is closer to the sink. To conduct the migration, $P_{n}^{\prime}$ sends a message migrate_req $\left(P_{n}^{\prime}\right)$ to $P_{n}$. On receiving the message, $P_{n}$ removes itself from the tree if it is a leaf, and sends an $a d d \_r e q\left(P_{n}^{\prime}\right)$ to its parent (denoted as $P_{i}$ ).

When $P_{i}$ receives the message, it checks if $P_{n}^{\prime}$ is in its spanning range. If it is still in the range, $P_{i}$ follows the procedure of adding a new proxy (as described in Section 4.2.2) to add $P_{n}^{\prime}$ to the tree rooted at $P_{i}$. Otherwise, it sends a message $a d d \_r e q\left(P_{n}^{\prime}\right)$ to its parent. The process continues until $P_{n}^{\prime}$ finally joins the tree.

\subsubsection{Source Movement-Triggered Tree Reconfigura- tion}

When a source becomes far away from its current proxy $(P), P$ should also be changed to another node $\left(P^{\prime}\right)$ which is closer to the source. $P^{\prime}$ becomes the new root of the proxy tree, and $P$ becomes its child. The change of root causes the other nodes in the tree to change their spanning ranges, and the information about the new spanning ranges is passed from the root to leaves as the sensing data flow. On receiving its new spanning range, each node $P_{n}$ checks its children one by one in a certain order (e.g., clockwise order), and decides whether the position of a child should be changed. Specifically, if a child $P_{n, k}$ becomes outside of the spanning range of $P_{n}$, a message rearrange_req $\left(P_{n, k}\right)$ is sent to its parent, which decides the new position of $P_{n, k}$ in the same way as described in Section 4.2.2. Otherwise, the position of $P_{n, k}$ is unchanged.

\section{Performance Evaluations}

We first use MATLAB to simulate the proposed centralized schemes and the distributed schemes, and compare their performance in terms of the average weight of proxy trees, without considering the tree reconfiguration overhead. After that, simulations based on NS2 are conducted in more practical scenarios to evaluate the performance of the proposed distributed schemes.

\subsection{Comparing the Centralized and the Dis- tributed Schemes}

The MATLAB-based simulations are conducted in the following settings: 516 (or 2064) nodes are uniformly distributed in a $500 \times 500 \mathrm{~m}^{2}\left(\right.$ or $\left.1000 \times 1000 \mathrm{~m}^{2}\right)$ square. One 
target and 10 sinks move randomly within the sensing region. Data are sent from the source (whose location is the same as the target) to the sinks every second. The proposed centralized schemes, ONMST and E-ONMST, and the distributed schemes, SP and SR, are simulated. We use the sum of the average tree weight as the metric to compare the performance of these schemes. In the simulations, each experiment lasts for $300 \mathrm{~s}$, and 60 experiments are conducted for each scheme. The average results of these experiments are shown in the figures.

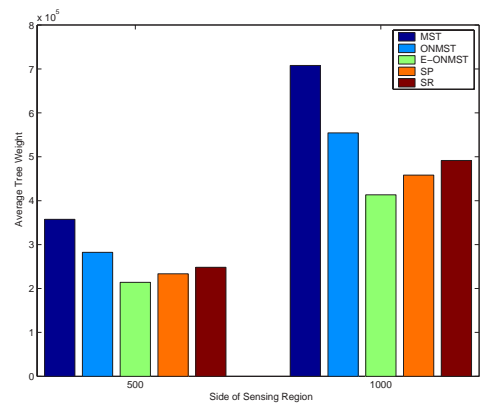

Figure 8. Comparing the tree weights of different schemes (average velocity $=2.5 \mathrm{~m} / \mathrm{s}$, localized reconfiguration interval $=1 \mathrm{~s}$ )

As shown in Figure 8, the average tree weight is the largest when the minimum spanning tree (MST) of the proxies is used for data dissemination. When the ONMST scheme is employed, the tree weight can be reduced by about $25 \%$, since ONMST can select some Steiner points to reduce the tree weight. The tree weight can be further reduced by about $20 \%$ when using the E-ONMST scheme, which can periodically optimize the tree reconfigured by ONMST. The shortest path-based (SP) scheme and the spanning range-based (SR) scheme have about $9 \%$ and $12 \%$ higher tree weight than the E-ONMST scheme, respectively. This is due to the reasons that they use less information to reconfigure the tree.

\subsection{Evaluating the Distributed Algorithms}

\subsubsection{Simulation Model}

In the NS2-based simulations, the IEEE 802.11 MAC layer protocol and the location-based GPSR routing algorithm are employed. We uniformly deploy 516 sensor nodes over a $500 \times 500 m^{2}$ field. Each sensor node has a communication range of $40 \mathrm{~m}$. One target and 10 sinks move randomly in the field, and the way-point model is used to simulate their movement. As a sink or a source (target) moves $80 \mathrm{~m}$ away from its current proxy, the sensor node closest to it is selected as the new proxy.

We evaluate the following metrics:
- Control message complexity: the number of control messages transmitted in the network.

- Data message complexity: the number of data messages transmitted in the network.

- Overall message complexity: the sum of the control message complexity and the data message complexity.

In the simulations, each experiment lasts for $300 \mathrm{~s}$, and 60 experiments are conducted for each scheme. The average results of these experiments are shown in the figures.

\subsubsection{Comparing SR and SP}

Figure 9 (a) shows that SR has smaller control message complexity than SP, which is due to the following reasons: As a sink (source) changes its proxy, SP needs to flood discover messages within a certain area to let the new proxy join the proxy tree. After that, the new proxy also has to exchange several messages with the tree nodes within the flooding area to select the appropriate parent node. However, when SR is used, only a few messages need to be sent, because the new proxy is usually still within the spanning range of the parent of the previous proxy. So it can immediately join the subtree rooted at the parent node. Even if the new proxy is out of the spanning range, a reconfiguration will be conducted in the smallest subtree that covers the new proxy, and the process will not cause many control messages.

Figure 9 (b) shows that SR has slightly larger data message complexity than SP. This phenomenon is consistent to that shown in Figure 8, which verifies that the shortest path heuristic is slightly better than the spanning range heuristic. However, as shown in Figure 9 (c), SR outperforms SP in terms of the overall message complexity.

\subsubsection{Impact of the Localized Reconfiguration (LR) Mechanism}

Figure 10 (a) shows that using the LR mechanism increases the control message complexity. Also, the control message complexity increases as the system parameter $\alpha$ decreases. This is due to the reason that the localized reconfiguration is conducted more frequently as $\alpha$ becomes smaller. However, as shown in Figure 10 (b), using the LR mechanism can decrease the data message complexity, and the data message complexity decreases as the system parameter $\alpha$ decreases. Also, when $\alpha$ is very small (e.g., 0.05), decreasing the parameter does not significantly decrease the data complexity. This is due to the reason that the node density is not large enough, and hence there may not exist a node at the optimal location to further minimize the cost, when $\alpha$ is too small. Figure 10 (c) shows that, with an appropriate parameter $\alpha$, 


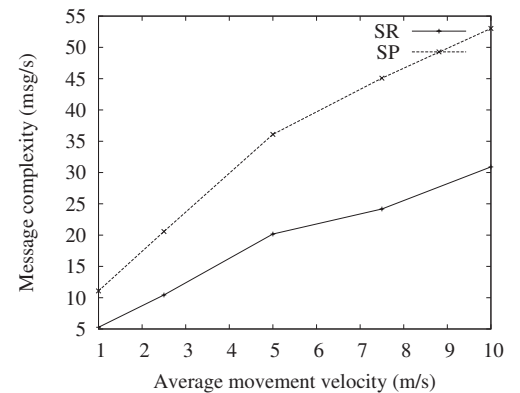

(a) Control message complexity

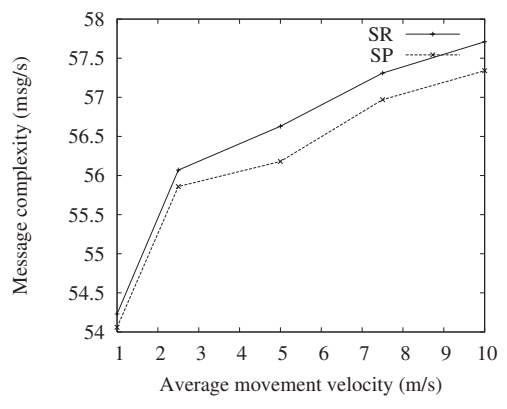

(b) Data message complexity

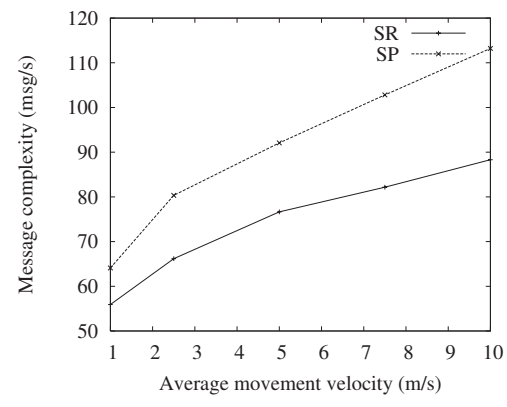

(c) Total message complexity

Figure 9. Comparing SR and SP

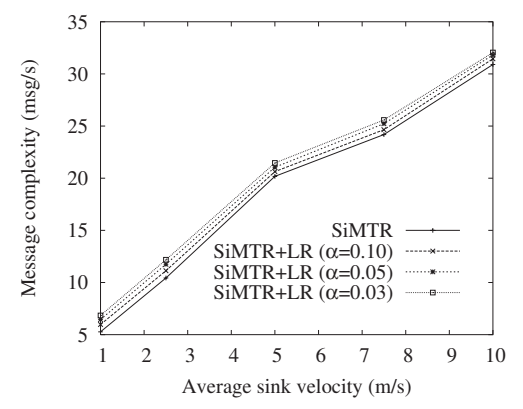

(a) Control message complexity

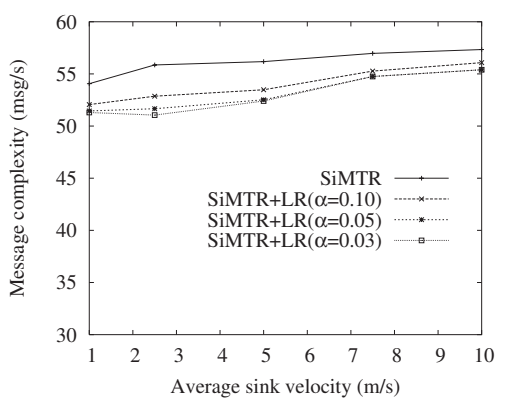

(b) Data message complexity

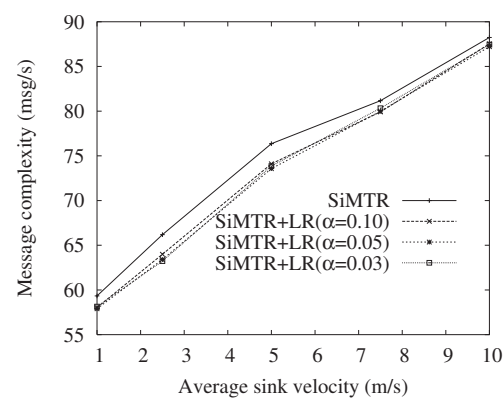

(c) Overall message complexity

\section{Figure 10. Impact of the localized reconfiguration mechanism ( SiMTR=Sink movement-initiated tree reconfiguration)}

using the LR mechanism can reduce the overall message complexity.

\subsubsection{Impact of the Source Movement-Initiated Tree Reconfiguration (SoMTR) Mechanism}

Figure 11 (a) shows that using the SoMTP mechanism increases the control message complexity, and the complexity increases as the source velocity increases. This phenomenon can be explained as follows: As the source moves, the source proxy changes accordingly. When the SoMTP mechanism is employed, changing the source proxy causes some nodes migrate from one branch to another. To conduct the reconfigurations, some control messages should be exchanged. Also, as the source velocity increases, the source proxy changes more frequently, which introduces more control messages.

Figure 11 (b) shows that using the SoMTP mechanism can reduce the data message complexity, which is due to the following reasons: If the SoMTP mechanism is not used, the tree is not reconfigured as the source proxy changes. Thus, data should be transmitted from the source to the previous proxy (root) before being transmitted to the sink prox- ies. However, when the SoMTP mechanism is used, the tree structure is optimized as the source proxy changes, and hence reduces the data dissemination cost.

Figure 11 (c) compares the overall message complexity when the SoMTP mechanism is used or not. As shown in the figure, as the source velocity increases, the SoMTP mechanism can significantly reduce the overall message complexity when the source velocity is not large. But the reduction becomes smaller when the source velocity is very large. The reasons can be found from Figure 11 (a) and (b). As the source velocity is small, the increment of the control message complexity is much slower than the reduction of the data message complexity. However, as the source velocity becomes very large, the increment of the control message complexity is similar to the reduction of the data message complexity.

\section{Conclusion}

In this paper, we addressed the problem of efficient dynamic multicasting in wireless sensor networks. We proposed a dynamic proxy tree-based framework, and focused on the issue of efficiently reconfiguring the proxy tree as 


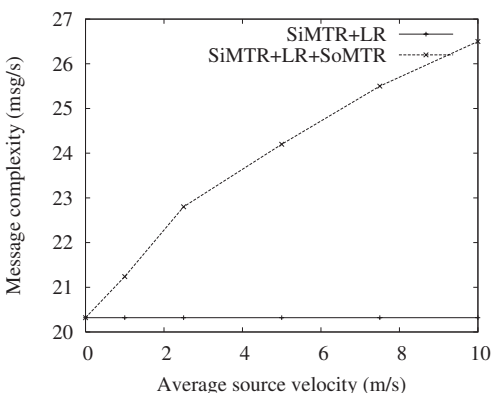

(a) Control message complexity

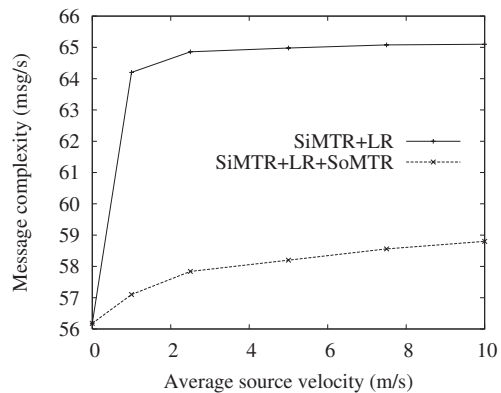

(b) Data message complexity

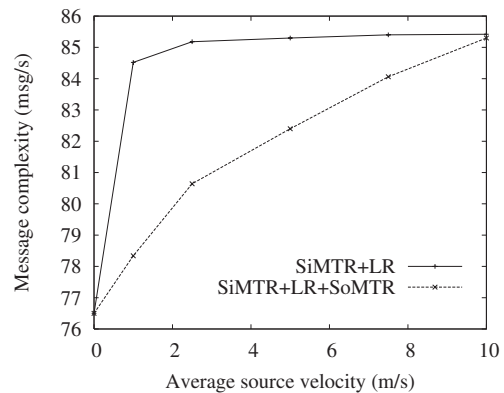

(c) Overall message complexity

Figure 11. Impact of the SoMTP mechanism (average sink velocity $=5.0 \mathrm{~m} / \mathrm{s}$ )

proxies frequently change from one node to another. The problem was modeled as on-line reconstructing a Steiner minimum tree in an Euclidean plane. Some centralized online schemes were proposed to solve the problem. Considering the strict energy constraints and the locality requirements in wireless sensor networks, we further proposed two distributed schemes, the shortest path-based (SP) scheme and the spanning range-based (SR) scheme. Extensive simulations were conducted to evaluate the proposed schemes. The results showed that the distributed schemes can achieve similar performance as the centralized schemes, and the SR scheme outperforms the SP scheme.

\section{References}

[1] "US Naval Observatory (USNO) GPS Operations," http://tycho.usno.navy.mil/gps.html, April 2001.

[2] I. Akyildiz, W. Su, Y. Sankarasubramaniam, and E.Cayirci, "Wireless Sensor Networks: A Survey," Computer Networks, vol. 38, no. 4, March 2002.

[3] Fred Bauer and Anujan Varma, "ARIES: A Rearrangeable Inexpensive Edge-Based On-Line Steiner Algorithm," IEEE Journal of Selected Areas in Communications, vol. 15, no. 3, pp. 382-397, 1997.

[4] P. Bose, P. Morin, I. Stojmenovic, and J. Urrutia, "Routing with Guaranteed Delivery in Ad Hoc Wireless Networks," Wireless Networks, vol. 7, no. 6, pp. 609-616, 2001.

[5] N. Bulusu, J. Heidemann, and D. Estrin, "GPS-less Low Cost Outdoor Location For Very Small Devices," IEEE Personal Communication, Special Issue on "Smart Space and Environments", October 2000.

[6] M. Chu, H. Haussecker and F. Zhao, "Scalable Informationdriven Sensor Querying and Routing for Ad Hoc Heterogeneous Sensor Networks," International Journal of High Performance Computing Applications, 2002.

[7] T. Cormen, C. Leiserson and R. Rivest, "Introduction to Algorithms," The MIT Press, pp. 514-543, 1990.

[8] A. Ghose, J. Grobklags and J. Chuang, "Resilient datacentric storage in wireless ad-hoc sensor networks," Proceedings the 4th International Conference on Mobile Data Management (MDM'03), pp. 45-62, 2003.

[9] B. Greenstein, D. Estrin, R. Govindan, S. Ratnasamy and S. Shenker, "DIFS: A Distributed Index for Features in Sensor Networks," First IEEE Ineternational Workshop on Sensor Network Protocols and Applications, May 2003.
[10] H. Laboid and H. Moustafa, "Source Routing-based Multicast Protocol (SRMP)," Internet Draft, 2001.

[11] W. Heinzelman, A. Chandrakasan, and H. Balakrishnan, "Energy-Efficient Communication Protocal for Wireless Microsensor network," Proc. of the Hawaii International Conference on System Sciences, January 2000.

[12] F. Hwang, D. Richards, and R. Winter, "The Steiner Tree Problem," Annals of Discrete Mathematics 53. Elsevier Science Publishers, 1992.

[13] C. Intanagonwiwat, R. Govindan, and D. Estrin, "Directed Diffusion: A Scalable and Robust Communication," MobiCOM '00, August 2000.

[14] A. Ivanov and A. Tuzhilin, "Minimal Networks," CRC Press, Inc., 1994.

[15] B. Karp and H. Kung, "GPSR: Greedy Perimeter Stateless Routing for Wireless Networks," The Sixth Aunual ACM/IEEE International Conference on Mobile Computing and Networking (Mobicom 2000), Aug. 2000.

[16] S. Lee, W. Su, and M. Gerla, "On-Demand Multicast Routing Protocol (ODMRP) for Ad Hoc Networks," IEEE ICNP'98, 1998.

[17] M. Liu, R. Talpade, A. Mcauley, and E. Bommaiah, "Ad Hoc Multicast Routing Protocol (AMroute)," UMD TechReport 99-8, 1999.

[18] N. Alon and Y. Azar, "On-line Steiner Trees in the Euclidean Plane," 2000.

[19] S. RatNasamy, B. karp, L. Yin, F. Yu, D. Estrin, R. Govindan, and S. Shenker, "GHT: A Geographic Hash Table for DataCentric Storage," ACM International Workshop on Wireless Sensor Networks and Applications, September 2002.

[20] J. Smith, D. Lee, and J. Liebman, "An O(nlogn) Heuristic for Steiner Minimal Tree Problems on the Euclidean Metric," Networks, pp. 11:12-29, 1981.

[21] F. Ye, H. Luo, J. Cheng, S. Lu, and L. Zhang, "A Two-Tier Data Dissemination Model for Large-scale Wireless Sensor Networks," ACM International Conference on Mobile Computing and Networking (MOBICOM'02), pp. 148-159, September 2002.

[22] W. Zhang and G. Cao, "DCTC: Dynamic Convoy TreeBased Collaboration for Target Trackin $\mathrm{g}$ in Sensor Networks," IEEE Transactions on Wireless Communication, in press, also available at: http://www.cse.psu.edu/ gcao.

[23] W. Zhang, G. Cao, and T. La Porta, "Data Dissemination with Ring-Based Index for Sensor Networks," IEEE International Conference on Network Protocol (ICNP), also available at: http://www.cse.psu.edu/ gcao, November 2003. 